\title{
Neutral Bilepton Boson Production in $p p$ Collisions from 3-3-1 Model
}

\author{
E. Ramirez Barreto, Y. A. Coutinho \\ Universidade Federal do Rio de Janeiro \\ 21941-972 - Rio de Janeiro, RJ, Brazil \\ J. Sá Borges \\ Universidade do Estado do Rio de Janeiro \\ 20550-013 - Rio de Janeiro, RJ, Brazil \\ (Received on 1 April, 2008)
}

\begin{abstract}
Our aim is to establish some signatures of the extra neutral gauge boson $X^{0}$, predicted in a version of the $S U(3)_{C} \times S U(3)_{L} \times U(1)_{X}$ model with right-handed neutrinos, by considering the process $p+p \longrightarrow X^{0}+X^{0 *}+$ $X$. In this work, we show some results concerning the LHC energy regime $(\sqrt{s}=14 \mathrm{TeV})$ and projected luminosity. Some distributions are shown and the $X^{0}$ width is calculated. We conclude that hadron colliders can show a clear signature for the existence of $X^{0}$ by comparing its production with $Z$ pair production.
\end{abstract}

Keywords: Beyond the Standard Model; New Gauge Bosons

\section{INTRODUCTION}

A peculiar feature of the Standard Model (SM) is that none of the gauge bosons carry baryon or lepton number. Many extensions of the SM predicted the existence of exotic particles carrying global quantum numbers, like leptoquarks and bileptons. The bileptons are defined as bosons carrying two units of lepton number and are present in $S U(15)$ grand unification theory and 3-3-1 models, for example.

In the 3-3-1 minimal version $[1,2]$, there are charged and doubly-charged bileptons and, on the other hand, the version with right-handed neutrinos [3] contains neutral and single-charged bileptons. In addition to these new gauge bosons, there are new quarks that carry two units of lepton number, they are called bileptoquarks. The production of charged bileptons at linear and hadron colliders was extensively studied and presents a unique signature, because they come from processes were total lepton number is conserved but the individual flavor lepton number is violated $[4,5]$.

A deep analysis of the extra neutral gauge boson $X^{0}$ pair production was not yet done. We intend to study the production of neutral gauge bileptons in hadron colliders. In this paper we show the total cross section and some distributions for the process $p+p \longrightarrow X^{0}+X^{0 *}+X$ calculated from $q \bar{q}$ at tree level. In the next section, we gives a brief review of the $3-3-1$ model with right-handed neutrinos. Section 3 is devoted to our results and discussion. In section 4 we presents the conclusions.

\section{THE MODEL}

The right-handed neutrino version ( $3-3-1 \mathrm{RHN})$, has in each leptonic triplet representation both the right- and lefthanded neutrino.

$$
\begin{aligned}
& \Psi_{a L}=\left(v_{a}, e_{a}, v_{a}^{C}\right)_{L}^{T} \sim(\mathbf{1}, \mathbf{3},-1 / 3), \\
& e_{a R} \sim(\mathbf{1}, \mathbf{1},-1),
\end{aligned}
$$

where $a=1,2,3$ is the generation index.
Two quark generations $(m=1,2)$ belong to anti-triplet and the other to triplet representation

$$
\begin{gathered}
Q_{m L}=\left(d_{m}^{\prime},-u_{m}^{\prime}, D_{m}^{\prime}\right)_{L}^{T} \sim\left(\mathbf{3}, \mathbf{3}^{*}, 0\right), \\
Q_{3 L}=\left(t^{\prime}, b^{\prime}, T^{\prime}\right)_{L}^{T} \sim(\mathbf{3}, \mathbf{3}, 1 / 3), \\
u_{a R}^{\prime} \sim(\mathbf{3}, \mathbf{1}, 2 / 3), d_{a R}^{\prime} \sim(\mathbf{3}, \mathbf{1},-1 / 3), \\
T_{R}^{\prime} \sim(\mathbf{3}, \mathbf{1}, 2 / 3), \quad D_{m R}^{\prime} \sim(\mathbf{3}, \mathbf{1},-1 / 3) .
\end{gathered}
$$

The new heavy quarks $D_{1}^{\prime}$ and $D_{2}^{\prime}$ carry $-\frac{1}{3}$ and $T^{\prime}$ carries $\frac{2}{3}$ units of positron charge.

The 3-3-1 model includes five new gauge bosons: a new neutral $\left(Z^{\prime}\right)$ two charged bileptons $\left(V^{ \pm}\right)$and two bileptons $\left(X^{0}\right)$ and $\left(X^{0 *}\right)$ with no electric charge.

One of the main features of the model comes from the relation between $Z^{\prime}$ and $V^{ \pm}$and $X^{0}$ masses:

$$
\frac{M_{V}}{M_{Z^{\prime}}} \simeq \frac{M_{X}}{M_{Z^{\prime}}} \simeq \frac{\sqrt{3-4 \sin ^{2} \theta_{W}}}{2 \cos \theta_{W}},
$$

From this relation we obtain $M_{X^{0}} \simeq 0.82 M_{Z^{\prime}}$ and so $Z^{\prime}$ is forbidden to decay into a bilepton pair $\left(V^{+} V^{-}\right.$or $\left.X^{0 *} X^{0}\right)$.

Three $S U(3)_{L}$ triplet $\eta, \rho$ and $\chi$ are necessary to generate masses for the particles and break the simmetry. These triplet develope vev's $v, u$ and $w$ respectively satisfying the relation $v^{2}+u^{2}=v_{W}^{2}$. We have considered in our calculations that [6]

$$
\frac{v_{W}^{2}}{w^{2}}<<1
$$

The interaction between the quark fields and the neutral bilepton $X^{0}$ is given by:

$$
\begin{aligned}
\mathcal{L}= & -\frac{g}{4 \sqrt{2}}\left\{\bar{t} \gamma^{\mu}\left(1-\gamma^{5}\right) T-\bar{D}_{m} \gamma^{\mu}\left(1-\gamma^{5}\right) d_{m}\right\} X_{\mu}^{0} \\
& + \text { H.c.. }
\end{aligned}
$$

As the exotic quark are very heavy, we discard their possible mixing with the ordinary physical quark fields and use the physical states identical to the symmetry states. 
TABLE I: The vector and axial-vector couplings between $Z$ and $Z^{\prime}$ with quarks $(u$ and $d$ ) in the $3-3-1$ RHN.

\begin{tabular}{|c|c|c|}
\hline & $g_{v}$ & $g_{a}$ \\
\hline$Z \bar{u} u$ & $\frac{1}{2}-\frac{4 \sin ^{2} \theta_{W}}{3}$ & $\frac{1}{2}$ \\
\hline$Z \bar{d} d$ & $-\frac{1}{2}+\frac{2 \sin ^{2} \theta_{W}}{3}$ & $-\frac{1}{2}$ \\
\hline$Z^{\prime} \bar{u} u$ & $\frac{3-8 \sin ^{2} \theta_{W}}{6 \sqrt{3-4 \sin ^{2} \theta_{W}}}$ & $\frac{1}{2 \sqrt{3-4 \sin ^{2} \theta_{W}}}$ \\
\hline$Z^{\prime} \bar{d} d$ & $\frac{3-2 \sin ^{2} \theta_{W}}{6 \sqrt{3-4 \sin ^{2} \theta_{W}}}$ & $\frac{1-2 \sin ^{2} \theta_{W}}{2 \sqrt{3-4 \sin ^{2} \theta_{W}}}$ \\
\hline
\end{tabular}

Keeping in mind this last observation, there is no flavor changing neutral currents (FCNC) coupled to $Z$ and $Z^{\prime}$, moreover we do not consider $Z-Z^{\prime}$ mixing. The neutral current Lagrangian is:

$$
\begin{aligned}
\mathcal{L}= & -\frac{g}{2 \cos \theta_{W}} \sum_{f}\left\{\bar{\Psi}_{f} \gamma^{\mu}\left(g_{v f}-g_{a f} \gamma^{5}\right) \Psi_{f} Z_{\mu}\right. \\
& \left.+\bar{\Psi}_{f} \gamma^{\mu}\left(g_{v f}^{\prime}-g_{a f}^{\prime} \gamma^{5}\right) \Psi_{f} Z_{\mu}^{\prime}\right\}+ \text { H.c. },
\end{aligned}
$$

where $f$ are quark fields and the vector and the axial couplings, $g_{v f}, g_{a f}, g_{v f}^{\prime}$ and $g_{a f}^{\prime}$, are given in the Table I, where we have considered the approximation between the vev's expressed in Eq. (5).

The trilinear couplings between $Z$ and $Z^{\prime}$ with $X^{0}$ and $X^{0 *}$ are respectively

$$
-\frac{g}{2 \cos \theta_{W}} \quad \text { and } \quad-\frac{g \sqrt{3-4 \sin ^{2} \theta_{W}}}{2 \cos \theta_{W}}
$$

\section{RESULTS}

The total width of the $X^{0}$ calculated from $\bar{q} Q$ and $v \vee$ channel contributions, where $Q$ represents the three bileptoquark and $v$ includes the three neutrino flavors. For $M_{X^{0}}=800,1000$ and $1200 \mathrm{GeV}$ are $1.54,6.70$ and $14.70 \mathrm{GeV}$. We use the CompHep package [7] in our calculations and assume $M_{Q}=600$ $\mathrm{GeV}$.

The main contributions for $X^{0}$ pair production in $p p$ collision depend on the initial quark $q$ charge. When $q=u$, only $Z$ and $Z^{\prime}$ via s-channel contribute and, on the other hand, when $q=d$ we have an additional t-channel heavy quark exchange

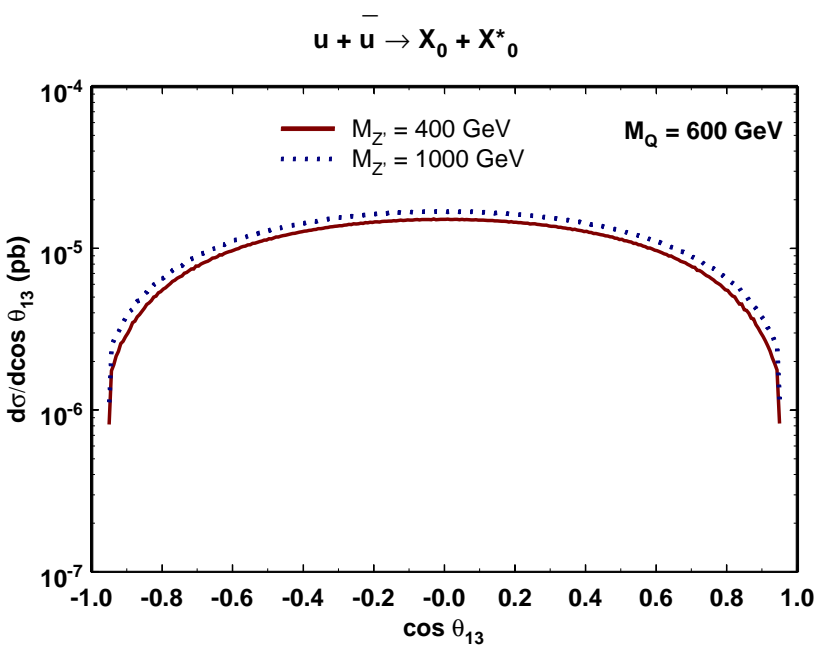

FIG. 1: $X^{0}$ angular distribution relative to the initial beam direction, considering $u \bar{u}$ channel for two different $Z^{\prime}$ masses.

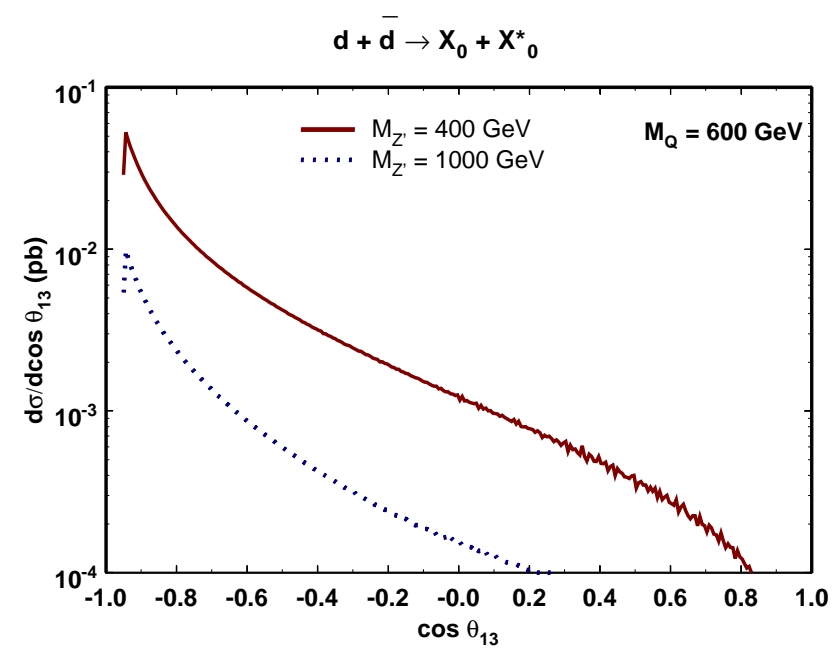

FIG. 2: $X^{0}$ angular distribution relative to the initial beam direction, considering $d \bar{d}$ channel for two different $Z^{\prime}$ masses.

contribution. We display, in Fig. 1 and in Fig. 2 respectively, the $X^{0}$ angular distribution relative to the initial beam direction for the elementary processes $u \bar{u}$ and $d \bar{d}$, adopting the following cuts on the final bileptons angle, rapidity and transverse momentum:

$$
|\cos \theta| \leq 0.95,|y| \leq 2.5, p_{t} \geq 50 \mathrm{GeV},
$$

The angular distribution shapes are different for $u$ and $d$ initial quarks. We observe the correct behavior of the total cross section for the elementary processes as shown the Fig. 3.

The total cross section and the distributions for the $p p$ process are obtained by the convolution of the elementary cross 


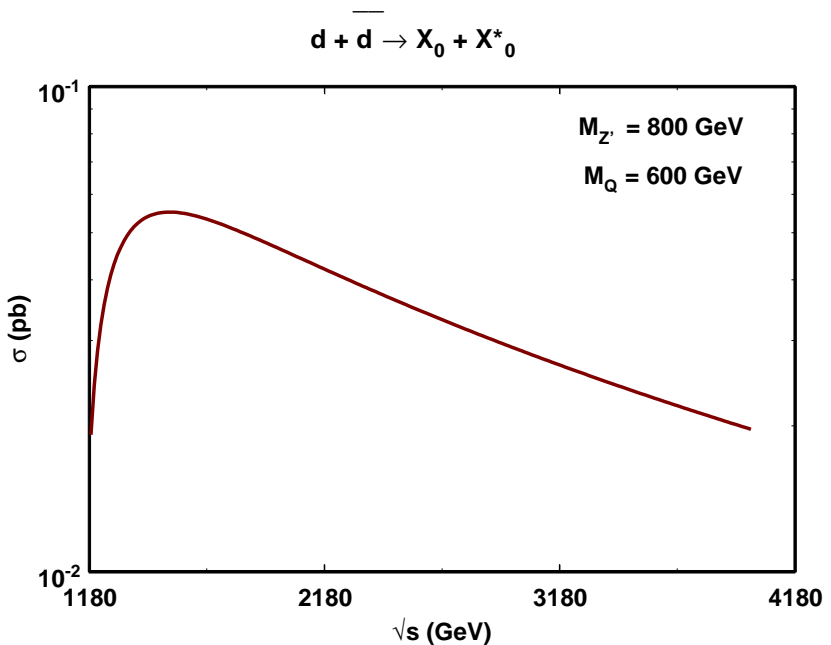

FIG. 3: Total cross section for $d \bar{d} \longrightarrow X^{0}+X^{0 *}$ vs. $\sqrt{s}$. The same behavior is observed for $u \bar{u} \longrightarrow X^{0}+X^{0 *}$.

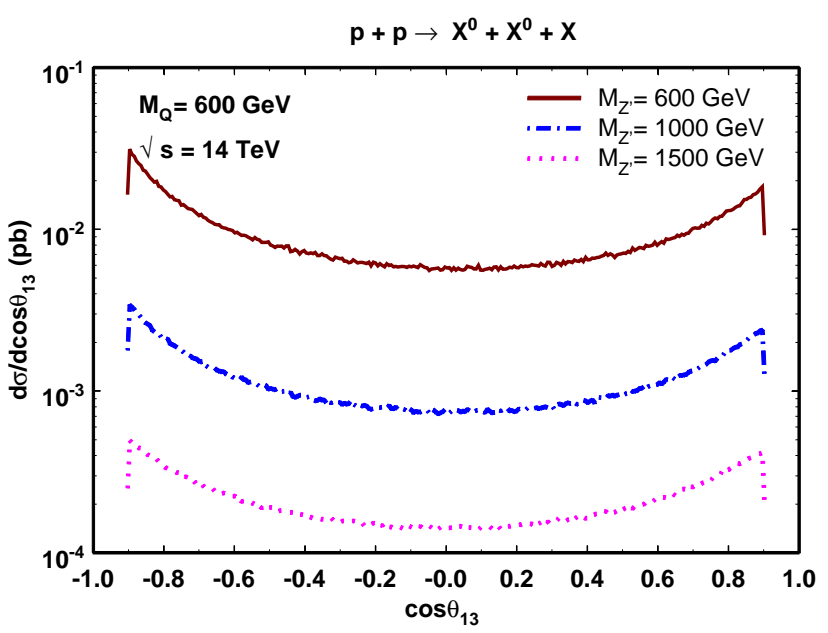

FIG. 4: $X^{0}$ angular distribution relative to the initial beam direction, for three different $Z^{\prime}$ masses, vs $\sqrt{s}$.

sections with the CTEQ661 [8] structure functions. The angular, invariant mass and rapidity distributions are displayed in Figs. 4, 5 and 6, respectively.

We conclude that the final $X^{0}$ angular distribution is almost flat. There is no preferential direction. We note that the total cross section for $M_{Q}=600 \mathrm{GeV}$ decreases when the $M_{Z^{\prime}}$ increases.

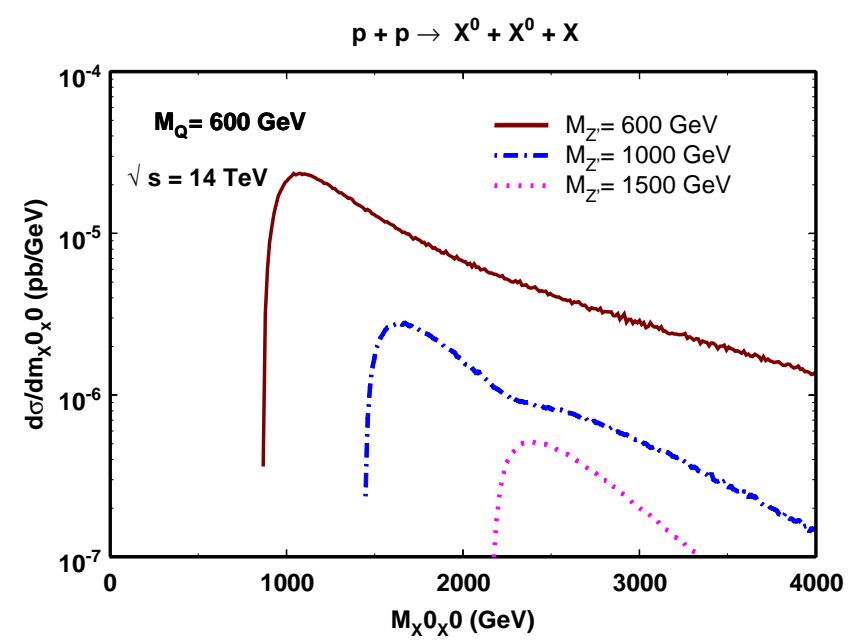

FIG. 5: $X^{0}$ pair invariant mass distribution, for three different $Z^{\prime}$ masses, vs. $\sqrt{s}$.

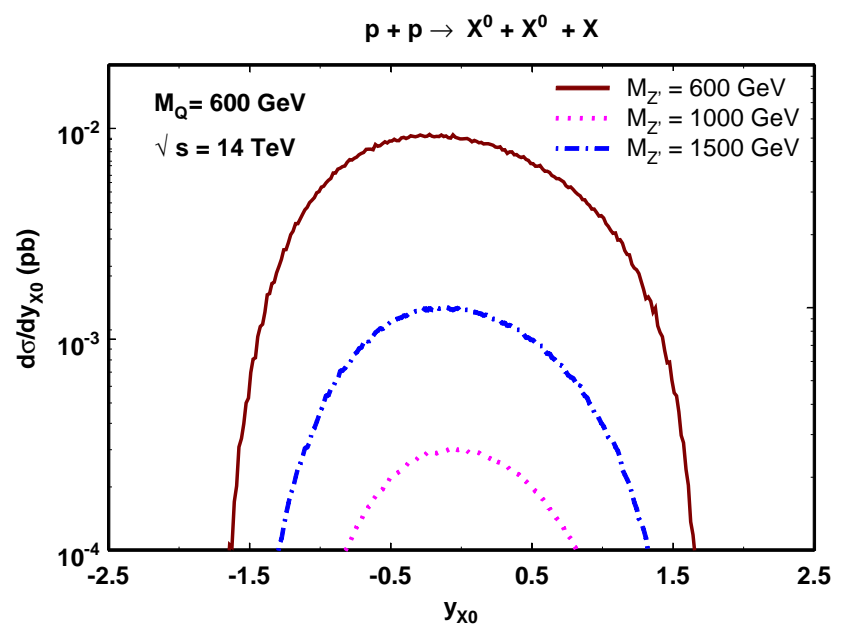

FIG. 6: $X^{0}$ rapidity distribution for three different $Z^{\prime}$ masses.

\section{CONCLUSIONS}

In this work we present preliminary results for the production of neutral bileptons, predicted for the $3-3-1$ RHN, from $p p$ collision. In a simple framework, without $Z-Z^{\prime}$ mixing and considering the absence of flavor changing neutral currents (FCNC), we have shown the bileptons distributions for the elementary processes and the results for $p p$ collisions.

For an annual luminosity at the $\operatorname{LHC}\left(\mathcal{L}=100 \mathrm{fb}^{-1}\right)$ we find around one thousand $X^{0}$ pairs produced per year. The study of the production of charged bileptons in $p p$ collisions from the minimal version of the $3-3-1$ model is being de- 
veloped and the preliminar results indicate a large number of events as well. The next step in our analysis is to consider the $X^{0}$ decay into two leptons in order to compare it with $Z$ decay. This comparison can possibly reveal a signature of the neutral bilepton production.

\section{Acknowledgments}

We acknowledge the financial support from CAPES (E.R.B.) and FAPERJ (Y.A.C.).
[1] F. Pisano and V. Pleitez, Phys. Rev. D 46, 410 (1992).

[2] P. H. Frampton, Phys. Rev. Lett. 69, 2889 (1992).

[3] J. C. Montero, F. Pisano, and V. Pleitez, Phys. Rev. D 47, 2918 (1993); R. Foot, H. N. Long, and T. A. Tran, Phys. Rev. D 50, R 34 (1994); Hoang Ngoc Long, Phys. Rev. D 53, 437 (1996); ibid 54, 4691 (1996); V. Pleitez, Phys. Rev. D 53, 514 (1996).

[4] B. Dion, T. Gregoire, D. London, L. Marleau, and H. Nadeau, Phys. Rev. D 59, 075006 (1999).
[5] E. Ramirez Barreto, Y. A. Coutinho, and J. Sá Borges, Phys. Lett. B 632, 675 (2006).

[6] Alex G. Dias, Thesis "O Problema da Violação CP Forte e o Limite Perturbativo em extensões $S U(3)_{C} \times S U(3)_{L} \times U(1)_{X}$ do Modelo Padrão", 2005.

[7] A. Pukhov et al., INP MSU 98-41/512, hep-ph/9908288.

[8] J. Pumplin et al., JHEP 207, 12 (2002). 